\title{
Hall Conductivity in the presence of repulsive magnetic impurities
}

\author{
Jean Desbois, Stéphane Ouvry and Christophe Texier
}

Laboratoire de Physique Théorique et Modèles Statistiques, 91406 Orsay Cédex, France.

\begin{abstract}
The Hall conductivity of disordered magnetic systems consisting of hard-core point vortices randomly dropped on the plane with a Poissonian distribution, has a behavior analogous to the one observed experimentally by R. J. Haug, R. R. Gerhardts, K. v. Klitzling and K. Ploog, with repulsive scatterers [1]. We also argue that models of homogeneous magnetic field with disordered potential, have necessarily vanishing Hall conductivities when their Hilbert space is restricted to a given Landau level subspace.
\end{abstract}

It is commonly believed [2] that in quantum Hall devices disorder plays a crucial role in the understanding of plateaus for the Hall conductivity as a function of $1 / B$ or $N\left(E_{F}\right)$, the number of electrons, at integer (or fractional) values in units of $e^{2} / h$. For an homogeneous $B$ field, the linear response of the system to a small electric field gives no hint of such a remarkable behavior since all the states are delocalized and have the same transverse conductivity which varies linearly with $1 / B$ or $N\left(E_{F}\right)$ (classical straight line). Disorder is needed to explain why some states (in fact most of them) are localized in broadened Landau levels, thus the plateaus in the Hall conductivity, around the classical line. However, R. J. Haug et al [1] reported the experimental observation of a shifted quantized Hall conductivity with respect to the classical line, when attractive or repulsive scaterrers are considered in the Hall sample. The data - Hall conductivity versus the filling factor- are shifted to the left when the scaterrers are repulsive, and to the right when they are attractive. The authors in [1] were able to reproduce qualitatively these shifts within a self-consistent T-matrix approximation computation. They also argued that two-dimensional disordered $\delta$ repulsive models projected in the lowest Landau level (LLL) of an external magnetic field [3], which exhibit an asymmetrically disorder induced broadened LLL, indeed favour this phenomenon. The root of the understanding lies in the fact that conducting states are not located anymore at the center of a symmetrically broadened Landau level, as it is the case for neutral scatterers, but in one side or the other of the now asymmetric density of states (DOS). On the theoretical side on the other hand, the question was asked in [3] about the way to compute exactly the average LLL Hall conductivity.

In the following, we study a model where the disorder is contained in the definition of the magnetic field itself. We consider 㭡 a gas of electrons coupled to hard-core point vortices, hereafter called magnetic impurities, carrying a flux $\phi=\alpha \phi_{o}\left(\phi_{o}=h / e\right.$ is the quantum flux) and randomly dropped on the plane according to a Poisson distribution, 
with Hamiltonian (in units $m_{e}=\hbar=1$ )

$$
H=\frac{1}{2}(\vec{p}-e \vec{A}(\vec{r}))^{2}-\sigma_{z} \frac{e}{2} B(\vec{r})
$$

The Aharonov-Bohm vector potential $e \vec{A}(\vec{r})=\alpha \sum_{i} \frac{\vec{k} \times\left(\vec{r}-\vec{r}_{i}\right)}{\left|\vec{r}-\vec{r}_{i}\right|^{2}}$ and the magnetic field $\vec{B}(\vec{r})=$ $\phi \sum_{i} \delta\left(\vec{r}-\vec{r}_{i}\right)$ depend on the configuration of the random positions $\vec{r}_{i}$ of the impurities. It is always possible to take $\alpha \in[0,1 / 2]$ because of the periodicity of period 1 in $\alpha$ and of the symmetry $\alpha \rightarrow-\alpha$. The spin assignation in (1) is needed to define in a non ambiguous way the model at hand. We choose $\sigma_{z}=-1$ which yields a short distance regularization for the impurities such that they are hard-core. It was shown in [⿴囗十 that two distinct spectral behaviors for the average DOS occur when $\alpha$ varies from $\alpha=1 / 2$ (i.e. big flux) and $\alpha \simeq 0$. When $\alpha$ is big, the electrons see the impurities individually, a maximum disordered situation with a free DOS but a depletion of states at origin of the spectrum. In contrast, for small value of $\alpha$, the inhomogeneities of the disordered magnetic field are less relevant, therefore a Landau like average DOS, with Landau oscillations, i.e. Landau levels separated by a mean Landau gap $e\langle B\rangle$ and broadened by disorder $(\langle B\rangle=\rho \phi$ is the mean magnetic field through the plane, $\rho$ is the mean impurity density - if $\rho$ is taken to be of the order of the density of current carriers $\rho=4.10^{15} \mathrm{~m}^{-2}$, one obtains, for $\alpha=1 / 2$, a mean magnetic field precisely in the experimental range of the Quantum Hall Effect $\langle B\rangle \simeq 10 T$ ). Thus, in the small $\alpha$ limit, the random magnetic impurity model has the required properties, i.e. an average magnetic field with disorder induced broadened Landau levels, to induce localization and eventually a quantized Hall conductivity.

The non unitary transformation $\left(\left\langle\omega_{c}\right\rangle=e\langle B\rangle / 2\right)$

$$
\psi=\mathrm{e}^{-\frac{1}{2}\left\langle\omega_{c}\right\rangle r^{2}} \prod_{i=1}^{N}\left|\vec{r}-\vec{r}_{i}\right|^{\alpha} \tilde{\psi}^{\prime}
$$

leads to the equivalent Hamiltonian

$$
\tilde{H}^{\prime}=\frac{1}{2} \Pi_{+}^{\langle\mathrm{L}\rangle} \Pi_{-}^{\langle\mathrm{L}\rangle}-\mathrm{i} \alpha(\Omega-\langle\Omega\rangle) \Pi_{-}^{\langle\mathrm{L}\rangle}
$$

where $\Omega=\sum_{i} \frac{1}{\bar{z}-\bar{z}_{i}}$, and $\Pi_{+}^{\langle\mathrm{L}\rangle}$ and $\Pi_{-}^{\langle\mathrm{L}\rangle}$ are the covariant Landau operators for the mean magnetic field. The Hamiltonian (3) has the simple structure of a Landau Hamiltonian for the mean magnetic field plus a disordered potential. One might consider that the Hall conductivity computation could be simplified if the mean magnetic field is sufficiently strong so that one can neglect couplings between Landau levels, or, more drastically, retain only the LLL. Remarkably enough [5], the Hamiltonian (3), when projected in the LLL of the mean magnetic field, precisely yields the repulsive $\delta$ impurity Hamiltonian [3] in the LLL of the mean magnetic field

$$
H=H_{\langle\mathrm{LLL}\rangle}+\lambda \sum \delta\left(\vec{r}-\vec{r}_{i}\right)
$$

with $\lambda=2 \pi \alpha$. So the question: What is the average Hall conductivity for the LLL Hamiltonian (4) and, more generally, for Hamiltonians of the type $H=H_{L}+V(\vec{r})$, where $V(\vec{r})$ is a disordered potential, when $H$ is restricted to the Hilbert space of a given Landau level of $H_{L}$ ? The answer is: In the linear response formalism, such a conductivity vanishes, implying that a non vanishing conductivity necessarily arises from couplings 
between different Landau levels. We insist here that restricting the Hilbert space to a given landau level and computing the conductivity in this given subspace should not be confused with the problem of evaluating the contribution of a given Landau level to the total conductivity. The Hamiltonian restricted to the $n$th Landau level is $H^{(n)}=$ $E_{n} P_{n}+P_{n} V P_{n}$ with $E_{n}=2 \omega_{c}(n+1 / 2)$ and $P_{n}$ the energy and the projection operator of the $n$th Landau level $P_{n}\left(z, z^{\prime}\right) \stackrel{\text { def }}{=}\left\langle z\left|P_{n}\right| z^{\prime}\right\rangle=\frac{\omega_{c}}{\pi} L_{n}\left(\omega_{c}\left|z-z^{\prime}\right|^{2}\right) \mathrm{e}^{-\frac{1}{2} \omega_{c}\left(\left|z-z^{\prime}\right|^{2}-z \bar{z}^{\prime}+\bar{z} z^{\prime}\right)}$ and $\Pi_{+}^{\mathrm{L}} P_{n}=\left(1-\delta_{n, 0}\right) P_{n-1} \Pi_{+}^{\mathrm{L}}$ (accordingly $\left.P_{n} \Pi_{-}^{\mathrm{L}}{ }^{\pi}=\left(1-\delta_{n, 0}\right) \Pi_{-}^{\mathrm{L}} P_{n-1}\right)$. The complex thermalized conductivity for one electron $\sigma_{\beta}^{-}(t) \equiv \sigma_{x x}(t)-i \sigma_{y x}(t)$ rewrites as [6]

$$
\sigma_{\beta}^{-(n)}(t)=\theta(t) \frac{\mathrm{i} e^{2}}{2 V Z_{\beta}^{(n)}} \int d z d \bar{z} d z^{\prime} d \bar{z}^{\prime}\left(G_{\beta-\mathrm{i} t}^{(n)}\left(z^{\prime}, z\right) \Pi_{-}^{L} G_{\mathrm{i} t}^{(n)}\left(z, z^{\prime}\right) z^{\prime}-(\mathrm{i} t \rightarrow \beta+\mathrm{i} t)\right)
$$

where the propagator $G_{\beta}^{(n)}\left(z, z^{\prime}\right)$ for $H^{(n)}$ is by definition

$$
G_{\beta}^{(n)}\left(z, z^{\prime}\right)=\left\langle z\left|P_{n} e^{-\beta H^{(n)}}\right| z^{\prime}\right\rangle
$$

The operator $\Pi_{-}^{\mathrm{L}}$ in (5) happens to be flanked by two projectors. Since $P_{n} \Pi_{-}^{\mathrm{L}} P_{n}=0$, then necessarily $\sigma_{\beta}^{-(n)}(t)=0$, implying that the Hall conductivity for a gas of non interacting electrons vanishes as well when restricted to a given Landau level.

It follows that the full Hamiltonian (3) is needed to get a non trivial information on the conductivity. First order perturbation theory (Fig.1) gives a behavior for the Hall conductivity [6] which is quite reminiscent of the experimental data in [1] for repulsive scatterers. Of course we do not pretend to describe precisely this particular experimental situation. Still, we would like to emphasize that an enhancement of the Hall conductivity does appear in the presence of repulsive magnetic impurities, and that this phenomenon can be obtained only if all Landau levels are considered.

Acknowledgments: S.O. would like to thank J. Bellissard for indicating [1].

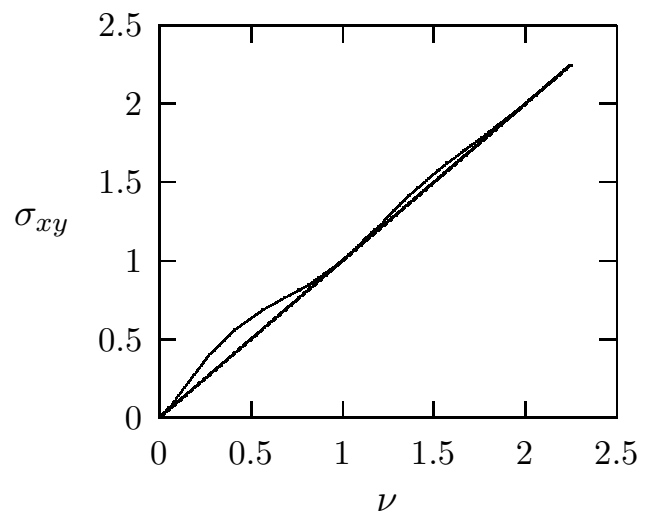

Figure 1: Hall conductivity in unit of $e^{2} / h$ of the random magnetic impurity model at first order in $\alpha$ for $\alpha=0.01$ as a function of the filling factor $\nu=\frac{N h}{V e\langle B\rangle}$; straight line $=$ classical result, full line $=$ perturbative result 


\section{References}

[1] R. J. Haug, R. R. Gerhardts, K. von Klitzing and K. Ploog, Effect of repulsive and attractive scattering centers on the magnetotransport properties of a two-dimensional electron gas, Phys. Rev. Lett. 59(12), 1349 (1987).

[2] M. Janssen, O. Viehweger, U. Fastenrath and J. Hadju, Introduction to the Theory of the Integer Quantum Hall Effect, ed. J. Hadju (VCH, Weinheim, 1994).

[3] E. Brézin, D. J. Gross and C. Itzykson, Density of states in the presence of a strong magnetic field and random impurities, Nucl. Phys. B [FS] 235, 24-44 (1984).

[4] J. Desbois, C. Furtlehner, and S. Ouvry, Random magnetic impurities and the Landau problem, Nucl. Phys. B [FS] 453, 759-776 (1995).

[5] J. Desbois, C. Furtlehner, and S. Ouvry, Random magnetic impurities and the $\delta$ impurity problem, J. Phys. I 6, 641-648 (1996).

[6] J. Desbois, S. Ouvry, and C. Texier, Hall conductivity for two-dimensional magnetic systems, Nucl. Phys. B [FS] 500, 486-510 (1997). 